\title{
Application of 3D bioprinting in the study of bacterial biofilms
}

\author{
Sergey Chapek $^{1, *}$, Sergey Golovin ${ }^{1}$, Michael Chikindas ${ }^{1,2}$, Svetlana Ponomareva ${ }^{1}$, Dmitry \\ Rudoy $^{1}$, and Anastasiya Olshevskaya ${ }^{1}$ \\ ${ }^{1}$ Don State Technical University, Gagarin Square,1, Rostov-on-Don, 344003 Russia \\ ${ }^{2}$ Rutgers State University, School of Environmental and Biological Sciences, 65 Dudley Road, New \\ Brunswick, NJ 08901, USA
}

\begin{abstract}
The article presents an overview of the main methods of obtaining biofilms in vitro, used in research. The technology of 3D-bioprinting is described - a new method in the modeling of bacterial biofilms, which solves one of the main problems in the study of biofilms- the heterogeneity of biomass, and opens up new opportunities for the study of various aspects of biofilm formation.
\end{abstract}

\section{Introduction}

There are two phenotypes of bacterial cultures: one is a single cell in a free-weighted state the so - called planktonic form of bacterial culture, the second one is a complex of bacterial aggregates, called biofilm. In the natural environment, up to $99 \%$ of all bacteria exist in the form of biofilms - organized communities which represent a three-dimensional structure that dynamically changes in space and time, consisting of actively functioning and resting cells enclosed in an intercellular matrix synthesized by them and formed at the interface between phases [1].

Despite the fact that the dental plaque biofilm is probably the first preparation containing bacteria that was studied by microscopy and described by A. van Leeuwenhoek in 1684 [2], the further development of microbiology, and, in particular, the work of R. Koch, which formed classical postulates [3], contributed to the study of pure cultures of bacteria that are phenotypically in planktonic form, and biofilms remained for a long time almost without any attention of researchers.

\section{Main part}

For the first time, the term "biofilm" is found in the work of M. I. Rakovskaya, describing bacterial conglomerates used in wastewater treatment [4].

The attention of medical microbiologists to biofilms became active due to the work of Costerton et al., [5,6,7,8] and the discovery of biofilm phenomenon role formation in chronic diseases of infectious etiology, bacterial persistence, implant-associated infections and antimicrobial resistance $[7,8,9,10]$.

\footnotetext{
*Corresponding author: s.chapek@sci.donstu.ru
} 
The discovery of the clinical significance of biofilms has made their study one of the trends in modern microbiology. The active study of biofilm formation phenomenon has led to the emergence of many methodological approaches which help to model this process.

One of the most important characteristics of biofilm and, at the same time, one of the main limitations of any experimental model, is its species composition. Biofilm is the most common naturally occurring phenotype of bacterial culture, which determines their multi-species composition. The methods for studying physiological parameters of pure bacterial cultures used for biofilms are convenient because they take into account only one variable; also they are relatively reproducible and methodically simple, in contrast to poly-species models. But from the point of view of "physiology" of the models, poly-specific cultures are more relevant.

The concept of microcosms-specific sets of microorganisms representing important physiological and ecological groups of microbial communities of the oral cavity [11], colon [12], aquatic ecosystems [13], etc. can be applied in the creation of poly-species models of different epitopes. The second important factor in modeling biofilms is the choice of cultivation method: periodic culture, semi-periodic or continuous. Classical methods of studying pure cultures in bacteriology are based on the paradigm of periodic cultivation, the conditions of which are rare in the natural environment. The majority of natural bacterial ecosystems can be considered as an example of continuous or semi-periodic cultivation.

The simplest model of biofilm can be considered an agar culture. Bacterial colonies on agar have many properties inherent in biofilms: they are located at the interface, contain a sufficient number of cells, there is a gradient of nutrients, gases and metabolites, etc. and Charaf et al. suggested using agar culture as a biofilm model to study the effects of antimicrobial drugs [14].

Another method of obtaining biofilm models is cultivation in 96-well microplates. This method is widely used, because it allows making a simple quantitative analysis of biofilms and effects of exposure to various substances on them. To implement this method, the test culture is inoculated into the wells of the tablet with a sterile culture medium (not only classical culture medium can be used, but also some physiological ones, for example, saliva or blood serum) and incubated under aerobic or anaerobic conditions with or without mixing, what depends on the experimental conditions and physiological characteristics of microorganisms.

The target indicator is the time and degree of bacterial cells adhesion to the bottom of the tablet cells after cultural fluid and non-adhered cells removal. Quantitative assessment of biofilm formation is carried out by staining a non-specific dye, for example, a crystalline violet, of biofilm formed at the interface of the phases. After that, the dye is dissolved in the specified volumes of ethanol and the colorimetric study is performed using microplate analyzers [15]. This method is useful, for example, when studying the effect of certain mutations on biofilm formation, the effects of antimicrobial agents, and the construction of growth curves in comparison with planktonic cultures when selecting optimal MIC.

Despite its simplicity and accessibility, this method has a number of significant drawbacks that affect the reproducibility of the results [16].

An important factor affecting biofilm formation in the microplate model is the property of the material from which the plates are made. The properties of the surface itself affect the nonspecific adhesion of bacterial cells to various surfaces [17]. Undoubtedly, when using tablets from different manufacturers, the adhesive properties of the plastic surface will vary [18].

The second factor affecting the reproducibility in this model is a sharp increase in the heterogeneity of biofilm biomass even in one plate, which contributes to the emergence of deviations when taking into account the results [19].

Mechanical damage and scratches, which inevitably occur when the experimenter works with the wells of the tablet (when selecting and applying liquids pipettes are used), also influence the reproducibility of the results in this method [19].

On the basis of the microplate method, universal systems, which are mass-produced, were developed and patented [20], [21]. The devices are a 96-well plate with a lid that has inserts 
complementary to the wells, which are a substrate for the formation of biofilm. This eliminates the fluctuations in the volume of biomass observed in the classical microplate method due to the deposition of cells under the gravity influence.

Also, by changing the material from which these inserts are made, it is possible to model biofilms on specific substrates, for example, using hydroxyapatite - the main mineral of tooth enamel and saliva as a growth medium, it is possible to model dental biofilm [22].

Despite the above-mentioned disadvantages of microplate methods, they remain widely demand and indispensable in screening studies for the evaluation of antibacterial drugs, coatings that prevent the formation of biofilms, disinfectants, selection of mutants etc.

When modeling biofilms, various dynamic systems are widely used: flow chambers and bioreactors. In 1981, McCoy and Robbins proposed an original device for studying biofilm formation in water supply systems [23]. Subsequently, this device was adapted for use in smallerscale laboratory experiments and became a standard model system for studying various aspects of biofilm formation under controlled conditions [24]. The modified Robbins device is a squaresection tube, equipped with ports located at the same distance from each other, in which the sampling instruments are placed.

An original device for modeling biofilm formation is a drop reactor [25], which allows obtaining biofilms on slide glasses, which is convenient for studying by various microscopy methods.

Various rotary bioreactors are used for modeling biofilms, for example, the so-called CDC (Centers for Disease Control and Prevention) biofilm reactor [26], which is mass-produced by BioSurface Technologies. Corp. (Bozeman, MT, USA). It is often used for producing reproducible biofilm samples under standard growth conditions to evaluate antibacterial agents and coatings. This bioreactor was included in the standard CDC biofilm study protocol [27], which contributed to its wide practical application. The reactor makes it possible to neutralize deviations that occur with changes in the cultivation temperature, nutrient concentration, and fluid shear stress [28].

The main drawback of these reactors is the limited number of individual strains that can be analyzed simultaneously and the increased risk of contamination of the reactor due to its design features.

Flow chamber systems are another way to model biofilms [29]. They are a set of closed optically transparent flow channels through which the nutrient medium with bacteria circulates. These systems, due to their relatively small size, allow conducting in vivo dynamic observation of biofilm formation using fluorescent or confocal laser scanning microscopy. However, sampling from most of these systems during dynamic observation is not possible, or there is a risk of contamination.

Microfluidic devices are a promising platform for studying biofilms. They are micrometersized flow capillaries in which biofilms can interact with hydrodynamic medium under mathematically predictable and finely controlled conditions that allow us to detect the influence of these hydrodynamic conditions on the development of biofilms [30, 31], as well as to investigate the effects of exposure to antibacterial substances[32]. Microfluidic devices can be made of a variety of materials: metal, glass, plastic, and polydimethylsiloxane (PDMS). The most commonly used material for microfluidic systems is PDMS. An important advantage of microfluidic systems is the convenience of in vivo dynamic visualization in real time and sampling [33, 34], as well as small volumes of reaction mixtures.

The main limitations in the use of microfluidic systems are the high cost of these platforms and peripheral devices and methodological complexity of working with them.

A common problem of all the above-mentioned methods of bacterial biofilms modeling is the high variability of the obtained biofilms parameters, and problems with the reproducibility of the results. This is partly explained by the fact that biofilm formation is mostly a stochastic 
process and methodological approaches used in biofilm modeling are not able to fully overcome this limitation.

A new method of modeling biofilms is 3D-bioprinting. This is a type of additive technologies, which are currently one of the fastest growing areas of engineering, actively integrating into tissue engineering and medicine [35, 36]. 3D bioprinting is the creation of three-dimensional cellular or tissue constructs using a robotic system in accordance with a given digital model.

Traditionally, 3D bioprinting is defined as a method of biofabrication using animal and / or plant cells [37]. However, different works that describe the use of bacteria in 3D bioprinting have become of current interest, although many authors in their works do not use the concept of "biofilm" in relation to aggregates of bacteria enclosed in a hydrogel scaffold [38,39,40,41]. Nevertheless, a number of studies show that bacteria in the composition of bio-printed scaffolds, with their sufficient amount to activate the mechanisms of biofilm formation - primarily quorum sensing - synthesize intercellular substance and form biofilms [42,43,44,45].

$3 \mathrm{D}$ bioprinting solves the main problem of biofilm modeling - the significant heterogeneity of biomass and offers a standardized approach to obtain reproducible biofilm models with a given architecture and size [44]. Bacteria in hydrogel scaffolds have increased metabolic activity, antimicrobial resistance, and plasmid stability compared to bacteria in 2D biofilm models [44]. This may indicate that 3D bio-printed bacterial biofilms more closely reflect the nature of bacterial growth in vivo compared to traditional 2D models and may expand methodological approaches for studying various aspects of biofilm formation and methods of controlling biofilms in the clinic [46].

One of the variants of the methodological approach in 3D-bioprinting of bacterial biofilms is schematically presented in the Figure 1.

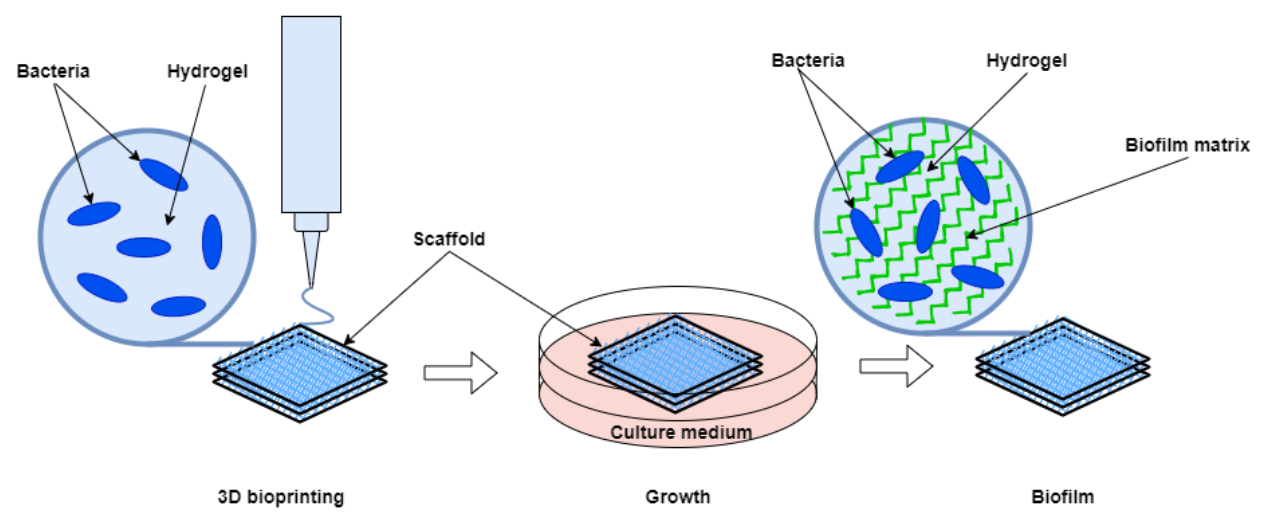

Fig 1. Diagram of the 3D biofilm bioprinting method.

In $3 \mathrm{D}$ extrusion bioprinting - the most widely used form of this technology - the biofilm biofabrication process involves creating a digital model, preparing hydrogel and introducing bacterial cells into it, directly scaffold 3D bioprinting and cultivation of scaffold on a dense or liquid nutrient medium until mature biofilm is formed. Further actions depend on the goals and objectives of a particular experiment. For example, the hydrogel that makes up scaffold can be removed in some cases [42] and a biofilm consisting of bacterial cells and its own extracellular matrix is obtained directly.

In addition to 3D extrusion bioprinting, biofabrication with bacterial cells is also shown using laser printing [47] and optogenetic methods [48].

However, in our opinion, it is $3 \mathrm{D}$ extrusion printing that makes it possible to obtain biofilms with a high degree of control over the concentration and distribution of biomass, thickness and size, as well as to form biofilms of a given configuration and architecture. 
Also, the use of 3D extrusion bioprinting allows forming large biofilms (limited only by the printing area of a particular bioprinter), which is impossible to achieve by other methods.

The use of eukaryotic cells of plants, animals and humans and components of their intercellular matrices in biofilm biofabrication can further expand the potential of this method, which will allow creating, for example, models of local infectious processes in vivo, or models of the microbiota of various epitopes (especially considering the possibility of printing multispecies biofilms) [49].

3D bio-printed biofilms can be an excellent model for testing antimicrobial agents and disinfectants and selecting the minimum inhibitory concentrations.

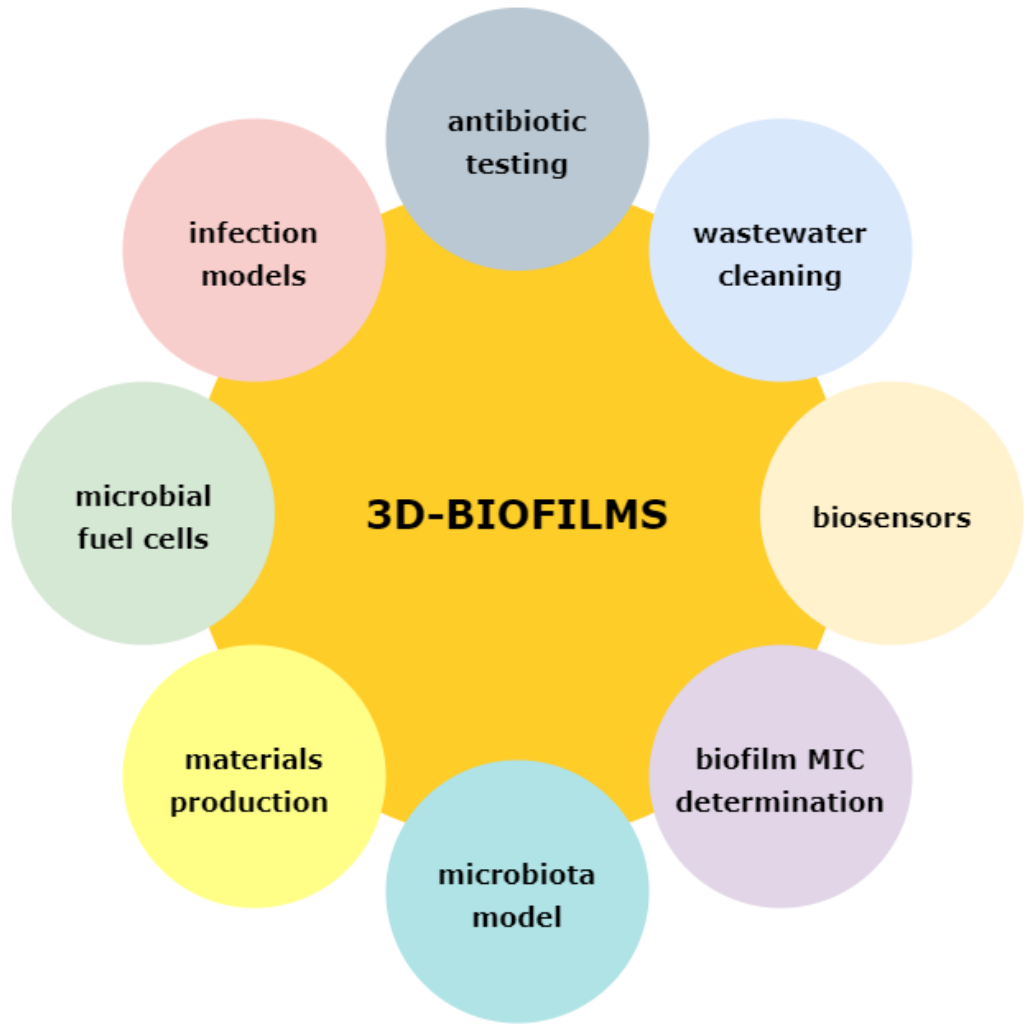

Fig. 2. Applications of biofilms.

\section{Discussion}

Currently, biofilms are already used to create microbial fuel cells [50], in wastewater treatment $[51,52]$, as biosensors $[53,54,54]$ and in biotechnology [56,57,58,59] (Fig. 1). The application of $3 \mathrm{D}$ bioprinting to form biofilms can provide completely new engineering applications for these objects.

\section{Acknowledgement}

Authors acknowledge the support of the Government of the Russian Federation (contract No. 075-15-2019-1880).

The work was carried out as the part of an initiative research project. 


\section{Refernces}

1. T. Bjarnsholt, APMIS 121, 1-58 (2013) doi:10.1111/apm.12099

2. A. van Leeuwenhoek, Philos Trans R Soc Lond B Biol Sci 14, 568-74 (1684)

3. R. Koch, Die Aetiologie der Tuberkulose. Berl Klin Wochenschr 15, 221-230 (1882)

4. M.I. Rakovskaya, M.F. Lazareva, Microbiology 30(4), 487-491 (1961)

5. J.W. Costerton, G.G. Geesey, K.J. Cheng, How bacteria stick. Sci Am 238, 86-95 (1978)

6. W.F. McCoy, J.D. Bryers, J. Robbins, J.W. Costerton, Canadian Journal of Microbiology 27(9), 910-917 (1981)doi:10.1139/m81-143

7. J.W. Costerton, P.S. Stewart, E.P. Greenberg, Science 284, 1318-22 (1999)

8. J.W. Costerton, The Biofilm Primer (Springer, New York, 2007)

9. N. Hoiby, T. Bjarnsholt, M. Givskov, S. Molin, O. Ciofu, Int J Antimicrob Agents 35, $322-$ $32(2010)$

10. A.S. McKee, A.S. McDermid, D.C. Ellwood, P.D. Marsh, Journal of Applied Bacteriology 59(3), 263-275 (1985) doi:10.1111/j.1365-2672.1985.tb01788.x

11. Newton, Cummings, Macfarlane, \& Macfarlane, Journal of Applied Microbiology 85(2), 372-380 (1998) doi:10.1046/j.1365-2672.1998.00522.x

12. C.M. Buswell, Y.M. Herlihy, P.D. Marsh et al., Journal of Applied Microbiology 83(4), 477-484 (1997) doi:10.1046/j.1365-2672.1997.00260.x

13. U.K. Charaf, S.L. Bakich, D.M. Falbo, Biofilms: The Good the Bad and the Ugly (Bioline, Cardiff, 1999)

14. G.A. O'Toole, Journal of Visualized Experiments 47 (2011) doi:10.3791/2437

15. K.N. Kragh, M. Alhede, M. Rybtke et al., Applied and Environmental Microbiology 84(5) (2017) doi:10.1128/AEM.02264-17

16. N.V. Seregina, T.V. Chestnova, V.A. Zherebtsova, V.A. Khromushin, Vestn. new medical technologies XV(3), 175-177 (2008)

17. K.N. Kragh, M. Alhede, M. Rybtke et al., Applied and Environmental Microbiology, 84(5) (2017) doi:10.1128/AEM.02264-17

18. K.N. Kragh, M. Alhede, L. Kvich, T. Bjarnsholt, Biofilm, 100006 (2019) doi:10.1016/j.bioflm.2019.100006

19. H. Ceri, M.E. Olson, C. Stremick et al., Journal of Clinical Microbiology 37(6), 1771-1776 (1999) doi:10.1128/JCM.37.6.1771-1776.1999

20. J. Azeredo, N.F. Azevedo, R. Briandet, et al., Critical Reviews in Microbiology 43(3), 313351 (2016) doi:10.1080/1040841x.2016.1208146

21. R.G. Ledder, T. Madhwani, P.K. Sreenivasan et al., Journal of Medical Microbiology 58(4), 482-491 (2009) doi:10.1099/jmm.0.006601-0

22. W.F. McCoy, J.D. Bryers, J. Robbins, J.W. Costerton, Canadian Journal of Microbiology 27(9), 910-917 (1981) doi:10.1139/m81-143

23. J.C. Nickel, I. Ruseska, J.B. Wright, J.W. Costerton, Antimicrobial Agents and Chemotherapy 27(4), 619-624 (1985) doi:10.1128/aac.27.4.619

24. D.M. Goeres, M.A. Hamilton, N.A. Beck et al., Nature Protocols 4(5), 783-788 (2009) doi:10.1038/nprot.2009.59

25. R.M. Donlan, J.A. Piede, C.D. Heyes et al., Applied and Environmental Microbiology 70(8), 4980-4988 (2004) doi:10.1128/aem.70.8.4980-4988.2004 
26. ASTM E2562-07, Standard Test Method for Quantification of Pseudomonas aeruginosa Biofilm Grown with High Shear and Continuous Flow using CDC Biofilm Reactor (ASTM International, West Conshohocken, PA, 2007) www.astm.org

27. D.M. Goeres, Microbiology 151(3), 757-762 (2005) doi:10.1099/mic.0.27709-0

28. T. Tolker-Nielsen, C. Sternberg, Pseudomonas Methods and Protocols, 615-629 (2014) doi:10.1007/978-1-4939-0473-0_47

29. V. Janakiraman, D. Englert, A. Jayaraman, H. Baskaran, Annals of Biomedical Engineering 37(6), 1206-1216 (2009) doi:10.1007/s10439-009-9671-8

30. J.-H. Lee, J.B. Kaplan, W.Y. Lee, Biomedical Microdevices 10(4), 489-498 (2008) doi:10.1007/s10544-007-9157-0

31. J. Terry, S. Neethirajan, Journal of Nanobiotechnology 12(1), 1 (2014) doi:10.1186/14773155-12-1

32. M.T. Meyer, V. Roy, W.E. Bentley, R. Ghodssi, Journal of Micromechanics and Microengineering 21(5), 054023 (2011) doi:10.1088/0960-1317/21/5/054023

33. M.R. Benoit, C.G. Conant, C. Ionescu-Zanetti et al., Applied and Environmental Microbiology 76(13), 4136-4142 (2010) doi:10.1128/aem.03065-09

34. W. Aljohani, M.W. Ullah, X. Zhang, G. Yang, International Journal of Biological Macromolecules 107, 261-275 (2018) doi:10.1016/j.ijbiomac.2017.08.171

35. Q. Yan, H. Dong, J. Su, et al., Engineering (2018) doi:10.1016/j.eng.2018.07.021

36. L. Moroni, T. Boland, J.A. Burdick et al., Trends in Biotechnology 36(4), 384-402 (2017) doi:10.1016/j.tibtech.2017.10.015

37. J.L. Connell, E.T. Ritschdorff, M. Whiteley, J.B. Shear, Proceedings of the National Academy of Sciences 110(46), 18380-18385 (2013) doi:10.1073/pnas.1309729110

38. M. Schaffner, P.A. Rühs, F. Coulter, S. Kilcher, A.R. Studart, Science Advances 3(12), eaao6804 (2017)doi:10.1126/sciadv.aao6804

39. B.A.E. Lehner, D.T. Schmieden, A.S. Meyer, ACS Synthetic Biology 6(7), 1124-1130 (2017) doi:10.1021/acssynbio.6b00395

40. L.M. González, N. Mukhitov, C.A. Voigt, Nat Chem Biol 16, 126-133 (2020). https://doi.org/10.1038/s41589-019-0412-5

41. D.T. Schmieden, S.J. Basalo Vázquez, H. Sangüesa et al., ACS Synthetic Biology 7(5), 1328-1337 (2018) doi:10.1021/acssynbio.7b00424

42. E.M. Spiesz, K. Yu, B.A.E. Lehner et al., Journal of Visualized Experiments 147 (2019) doi:10.3791/59477 https://doi.org/10.3791/59477

43. E. Ning, G. Turnbull, J. Clarke et al., Biofabrication (2019) doi:10.1088/1758-5090/ab37a0

44. J. Huang, S. Liu, C. Zhang, et al., Nature Chemical Biology (2018) doi:10.1038/s41589018-0169-2

45. S. Kyle, Trends in Biotechnology 36(4), 340-341 (2018) doi:10.1016/j.tibtech.2018.01.010

46. M. Rabbani, Y. Dalman, Bioprinting e00099 (2020) doi:10.1016/j.bprint.2020.e00099

47. Y. Huang, A. Xia, G. Yang, F. Jin, ACS Synthetic Biology 7(5), 1195-1200 (2018) doi:10.1021/acssynbio.8b00003

48. E. Saygili, A.A. Dogan-Gurbuz, O. Yesil-Celiktas, M.S. Draz, Bioprinting e00071 (2019) doi:10.1016/j.bprint.2019.e00071

49. M.J. Angelaalincy, R. Navanietha Krishnaraj, G. Shakambari et al., Frontiers in Energy Research 6 (2018) doi:10.3389/fenrg.2018.00063 
50. A. Di Biase, M.S. Kowalski, T.R. Devlin, J.A. Oleszkiewicz, Journal of Environmental Management 247, 849-866 (2019) doi:10.1016/j.jenvman.2019.06.053

51. H. Huang, C. Peng, P. Peng et al., Applied Microbiology and Biotechnology (2018) doi:10.1007/s00253-018-9511-6

52. R. Carafa, N.E. Lorenzo, J.S. Llopart, V. Kumar, M. Schuhmacher, Aquatic Toxicology 231, 105732 (2021) DOI: https://doi.org/10.1016/j.aquatox.2020.105732

53. H. Yang, M. Zhou, M. Liu et al., Biotechnology Letters 37(12), 2357-2364 (2015) doi:10.1007/s10529-015-1929-7

54. X. Qi, P.-P. Liu, P. Liang et al., Biosensors and Bioelectronics, 111500 (2019) doi:10.1016/j.bios.2019.111500

55. M.B. Cassidy, H. Lee, J.T. Trevors, Journal of Industrial Microbiology 16(2), 79-101 (1996) doi:10.1007/bf01570068

56. S. Balasubramanian, M.-E. Aubin-Tam, A.S. Meyer, ACS Synthetic Biology 8(7), 1564 1567 (2019) doi:10.1021/acssynbio.9b00192

57. A. Majerle, D.T. Schmieden, R. Jerala, A.S. Meyer, Biochemistry (2019) doi:10.1021/acs.biochem.8b00922

58. M. Mukherjee, B. Cao, Microbial Biotechnology (2020) doi:10.1111/1751-7915.13715

59. K.L. Rana, D. Kour, A.N. Yadav et al., Microbial Biofilms, 221-265 (2020) doi:10.1016/B978-0-444-64279-0.00016-5 